Available online at www.sciencedirect.com

www.cya.unam.mx/index.php/cya

Contaduría y Administración 60 (S2) 250-271

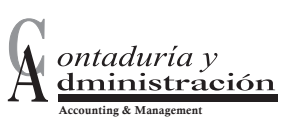

www.contaduriayadministracionunam.mx/

\title{
La reserva federal, la crisis y la política monetaria no convencional
}

\author{
The federal reserve, the crisis and unconventional \\ monetary policy
}

\section{Ignacio Perrotini Hernández}

Universidad Nacional Autónoma de México, Facultad de Economía, México

Recibido el 23 de septiembre de 2015; aceptado el 27 de octubre de2015

Disponible en internet el 20 de noviembre de 2015

\section{Resumen}

El artículo discute los fundamentos y los límites de la política monetaria convencional que estuvo vigente hasta la Gran Recesión, se analizan los elementos principales de la política monetaria no convencional (política de tasa de interés cero, flexibilización cuantitativa y forward guidance) implementada por la Fed desde el inicio de la Gran Recesión y concluimos que la nueva política monetaria no ha inducido una senda de crecimiento sostenido.

Derechos Reservados (C) 2015 Universidad Nacional Autónoma de México, Facultad de Contaduría y Administración.

Este es un artículo de acceso abierto distribuido bajo los términos de la Licencia Creative Commons CC BY-NC-ND 4.0.

Palabras clave: Tasa de interés; Política monetaria; Crisis financiera; Crecimiento Clasificación JEL: E43, E52, G01, O40.

Correo electrónico:iph@unam.mx.

La revisión por pares es responsabilidad de la Universidad Nacional Autónoma de México.

http://dx.doi.org/10.1016/j.cya.2015.11.001

0186-1042/Derechos Reservados (C) 2015 Universidad Nacional Autónoma de México, Facultad de Contaduría y Administración. Este es un artículo de acceso abierto distribuido bajo los términos de la Licencia Creative Commons CC BY-NC-ND 4.0. 


\begin{abstract}
Conventional and non conventional monetary policy frameworks followed by the Federal Reserve, prior and after the Great Recession, respectively, are dealt with in this paper. It is also shown that quantitative or credit easing, forward guidance and the zero interest rate monetary policy, all part and parcel of the Fed's new monetary policy approach, have failed to restore long-run sustainable growth.

All Rights Reserved (C) 2015 Universidad Nacional Autónoma de México, Facultad de Contaduría y Administración.

This is an open access item distributed under the Creative Commons CC License BY-NCND 4.0.

Keywords: Interest rate; Monetary policy; Financial crisis; Growth

JEL Classification: E43, E52, G01, O40.

So, as of December 2008, conventional monetary policy was exhausted. We could not cut the federal funds rate any further. And yet, the economy clearly needed additional support. Into 2009, the economy was still contracting at a rapid rate. We needed something else to support recovery, and so we turned to less conventional monetary policy.
\end{abstract}

Ben S. Bernanke (2013, p.102).

\title{
Introducción
}

El Federal Open Market Committee (FOMC), corpus de la Reserva Federal (Fed) encargado de conducir la política monetaria de los EE.UU., decidió una vez más no incrementar la tasa de interés de los fondos federales en su reunión del 17 de septiembre de 2015. La tasa de interés de los fondos federales $\left(i_{f f}\right)$ es el instrumento de la política monetaria de la Fed; el FOMC la ha mantenido en 0\%$0.25 \%$ (rango conocido como límite 0 de la tasa de interés nominal) desde el 16 de diciembre de 2008.

La actual política de tasa de interés cero (PTIC) constituye la primera ocasión en la historia que la Fed sostiene esta posición durante casi siete años (diciembre 2008-septiembre 2015). La PTIC es parte importante de la estrategia de la Fed para apoyar a la economía estadounidense en la fase de débil recuperación posterior a la Gran Recesión de 2007-2009 que indujo cambios significativos en la política monetaria.

La reciente noticia de Janet Yellen, gobernadora de la Fed, de que no habrá «lift-off» (aumento) de la tasa de interés de los fondos federales significa: en primer lugar, que las expectativas de crecimiento económico no son halagüeñas, pues EE.UU., Japón y Europa actualmente experimentan una recesión de hoja de ba- 
lance (Koo, 2015) después de haber acumulado cuantiosas deudas durante el auge previo a la crisis financiera; en segundo, que debido a la frágil recuperación económica y al temor al riesgo de deflación, es improbable que el FOMC aumente la $i_{f f}$ antes de 2018; y en tercer término, significa que el retorno a la política monetaria convencional o «normal» es incierto.

El hecho de que la Fed ha recurrido a la política monetaria no convencional durante 2008-2015 ${ }^{1}$ para contener el pánico financiero, evitar el riesgo de deflación y estimular la actividad económica ante la endeble recuperación del producto y el empleo, pone en tela de juicio la efectividad de la política monetaria de objetivo de inflación -en particular la idea de que el manejo de la tasa de interés garantiza la estabilidad de precios y la estabilidad financiera simultáneamente, tal como sostienen autores de la nueva síntesis neoclásica (cf. Taylor, 1993; Svensson, 1998; Bernanke et al., 1999; Woodford, 2003; Setterfield, 2005; Goodhart, 2009; Capraro y Perrotini, 2012; García y Perrotini, 2014).

El propósito principal del presente artículo es analizar la política monetaria no convencional (PMNC) de la Fed durante la post-Gran Recesión y discutir su viabilidad en una economía que confronta el límite cero de la tasa de interés nominal; en este artículo sostenemos que la PMNC no ha contribuido a estimular el crecimiento de la economía de los EE.UU. Para probar esta hipótesis, aplicamos las metodologías del análisis de cointegración, pruebas de raíces unitarias y un modelo autorregresivo de rezagos distribuidos (ARDL por sus siglas en inglés); los datos del análisis empírico comprenden series mensuales del periodo enero 2008-Junio 2015 de EE.UU.

Además de esta introducción, el artículo se compone de una segunda parte en que presentamos los fundamentos y los límites de la política monetaria convencional que estuvo vigente hasta el surgimiento de la Gran Recesión, política a la cual la Fed pretende retornar cuando la economía regrese a la senda de expansión sostenida (cf. Board of Governors of the Federal Reserve System, 2014). La tercera parte analiza y discute los elementos principales de la PMNC implementada por la Fed durante 2008-2015; en la cuarta parte presentamos algunos hechos estilizados del comportamiento de variables (crecimiento, inflación y el índice del mercado de valores) relevantes en la interacción entre la PMNC y la macroeconomía de EE.UU. La quinta parte contiene el análisis empírico y los resultados de la estimación econométrica que contrasta con evidencia estadística nuestra hipótesis; la última parte es la conclusión.

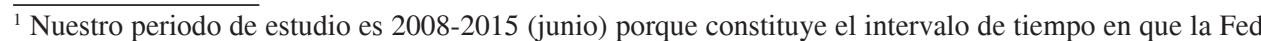
ha utilizado la hoja de balance y la política de flexibilización cuantitativa como marco de política monetaria «no convencional».
} 


\section{Los límites de la política monetaria convencional y la crisis}

En condiciones «normales» del ciclo económico, los bancos centrales en general y la Fed en particular realizan su política monetaria con base en el control de la tasa de interés nominal de corto plazo. La Fed influye en el curso de la economía mediante alzas y descensos de la $i_{f f}$, teniendo el objetivo dual de estabilidad de precios (inflación baja y estable) y máximo empleo. ${ }^{2}$ El marco general de la política monetaria convencional es el de metas de inflación ${ }^{3}$ y el instrumento que regula los precios es la tasa de interés $i_{f f}$ con base en la cual la Fed ofrece fondos «overnight» al mercado interbancario o a los bancos.

La conducta de la Fed puede expresarse mediante la regla de Taylor (Taylor, 1993):

$$
\hat{\imath}_{t}=\bar{\imath}_{t}+\gamma_{\pi}\left(\pi_{t}-\pi^{*}\right)+\gamma_{y}\left(y_{t}-y^{*}\right)
$$

$\hat{\imath}_{t}$ denota las fluctuaciones de la tasa de interés, el instrumento de la política monetaria del banco central, $\bar{l}_{t}$ es un intercepto exógeno que captura cambios en la meta de inflación y en el término de error, $\pi_{t}$ y $\pi^{*}$ representan la inflación observada y el objetivo de inflación (2\%) respectivamente, $y_{t}$ y $y^{*}$ denotan, respectivamente, el valor del producto observado y del producto potencial consistente con $\pi^{*} ; \gamma_{\pi}$ y $\gamma_{y}$ son coeficientes no negativos, los cuales, junto con $\pi^{*}$, son coeficientes de política constantes; $\gamma_{\pi}$ y $\gamma_{y}$ miden la reacción de la política monetaria ante desviaciones de $\pi_{t}$ y de $y_{t}$ vis-à-vis $\pi^{*}$ y $y^{*}$ respectivamente.

La ecuación (1) maximiza el bienestar del consumidor. Si $\gamma_{\pi}>1$, el banco central incrementa la tasa de interés real ante un aumento en la inflación para estabilizar los precios; si $\gamma_{\pi}<1$, el banco central puede no incrementar la tasa de interés nominal en proporción directa al aumento de la inflación, con lo cual disminuirá la tasa de interés real. El banco central sigue un modelo de objetivo de inflación «puro» cuando $\gamma_{y}=0$. La lógica del modelo de la regla de Taylor consiste en que $y_{t}=y^{*}$ significa que la brecha de producto es nula y que el producto potencial está determinado por la tasa natural de interés $\left(r_{t}^{n}\right)$. Las fluctuaciones de la tasa de interés nominal que el banco central utiliza como instrumento para alcanzar la meta

\footnotetext{
${ }^{2}$ El objetivo de máximo empleo no es necesariamente el pleno empleo de la fuerza de trabajo, sino más bien una meta de desempleo que la Fed establece ex ante de acuerdo con las condiciones específicas de la inflación, los mercados financieros y la actividad económica general (cf. Bernanke $(2012,2013)$.

${ }^{3}$ La inflación relevante en este análisis se refiere al índice de precios al consumidor. La Fed se ha enfocado históricamente en la inflación subyacente medida por los gastos de consumo personal; la inflación subyacente se mantiene por debajo de la meta de $2 \%$ establecida con la eclosión de la Gran Recesión. El FOMC aumentó el techo de la meta de inflación a 2.5\% en su reunión de diciembre de 2012, mientras a la par mantenía la tasa de interés de los fondos federales en su límite cero e implementaba medidas no convencionales como flexibilización monetaria inundando de liquidez el mercado. En reuniones posteriores, el FOMC volvió a la meta de inflación de $2 \%$.
} 
de inflación están determinadas por la tasa natural de interés y por las expectativas de inflación de los agentes económicos $\left(E_{t} \pi_{t+1}\right)$. Entonces, la función de reacción del banco central es como sigue:

$$
\hat{\imath}_{t}=\hat{r}_{t}^{n}+E_{t} \pi_{t+1}
$$

Si el banco central consigue la meta de inflación mediante esta regla de política monetaria convencional, entonces la economía converge hacia la tasa natural de interés y de producto potencial. A partir de la ecuación (2) podemos obtener la ecuación de Fisher:

$$
\left(1+\hat{r}_{t}^{n}\right)=\frac{1+\hat{\imath}_{t}}{1+\pi_{t}}
$$

En equilibrio, la tasa de interés nominal es igual a la suma de la tasa de interés real (o tasa de interés natural en el largo plazo) más la tasa de inflación:

$$
i=r+\pi
$$

Cuando el banco central logra la meta de inflación, $\pi=0$ y entonces $i=r$. En suma, la regla de Taylor no es sino una variante de la ecuación de Fisher (1907). Este es el modelo de inflación que la Fed siguió desde el periodo de la llamada Gran Moderación hasta la explosión de la crisis financiera de 2007-2009.

La primacía de la política monetaria en el control del ciclo económico, la demanda agregada y los precios se justifica en este paradigma porque mediante la manipulación de la $i_{f f}$ es posible hacer converger el producto y la tasa de interés real con sus valores naturales, es decir, obtener la convergencia entre estabilidad nominal y estabilidad real (Pérez Caldentey, 2015, p. 45).

Una condición crucial para el funcionamiento del modelo de política monetaria convencional es que la tasa de interés nominal no sea negativa. Esto se debe a que los agentes económicos en cualquier momento pueden preferir la liquidez absoluta cuando el rendimiento es cero (Keynes, [1936] 1964). El límite cero de la tasa de interés nominal restringe la efectividad de la política monetaria convencional. Este es el caso de la trampa de liquidez, situación en la que, dado un rango de ingreso, típicamente un aumento en la oferta monetaria no reducirá más la tasa de interés (Minsky, 1986, p. 133). Los problemas de la trampa de liquidez se presentan con mayor frenesí en la fase posterior a una gran depresión o a una crisis financiera (Minsky, 1975, p. 37), como lo constatan la evolución de la inflación, la tasa de interés y el estancamiento de la economía norteamericana desde que concluyó la Gran Recesión oficialmente en junio de 2009. De hecho, la tasa de crecimiento del producto interno bruto (PIB) ha sido inferior a 3\% en todos los años desde 2006, 
razón por la cual el Bureau of Economic Analysis afirma que el periodo 2006-2015 es el lapso más largo de crecimiento inferior a 3\% desde los años 1930.

Antes de la crisis financiera el instrumento efectivo de política normal era el control de la $i_{f f}$. Entre 2004 y agosto de 2007, la Fed la aumentó de 1\% a 5.25\% con el fin de abandonar la laxitud monetaria usada para combatir la recesión de 2001 y regresar a una posición de política monetaria «normal». Pero la explosión del mercado de hipotecas subprime en agosto de 2007 y el pánico financiero subsecuente, obligaron al FOMC a reducir la $i_{f f}$, primero, a 2\% en la primavera de 2008 y, posteriormente, a un rango de $0 \%-0.25 \%$, con lo cual la Fed comenzó a hacer política monetaria con una $i_{f f}$ que se encuentra en el límite cero de la tasa de interés (Bernanke, 2012).

En la literatura este problema se ha discutido como el caso en que la política monetaria del banco central es impotente para afectar el nivel de empleo y del producto. La situación actual de la economía norteamericana muestra que a fines de 2008 «la política monetaria convencional estaba agotada» (Bernanke, 2013). Así, la Fed adoptó la alternativa de la PMNC. Una $i_{f f}$ igual a 0\%-0.25\% implica, por una parte, que la Fed ha perdido su instrumento de política monetaria convencional más poderoso $\mathrm{y}$, por otra, que se obtura la conectividad entre la $i_{f f} \mathrm{y}$ las tasas de interés de mercado: es imposible reducir la $i_{f f}$ a valores negativos para estimular a la economía, y sin embargo la contumacia de la crisis requería tasas de interés negativas a pesar de que de facto esto no es posible.

Bernanke (2013) admitió que la política monetaria convencional fue exitosa en lo concerniente al objetivo de estabilidad de precios, pero fracasó en impedir burbujas financieras y en conseguir la estabilidad financiera. La inestabilidad de los mercados financieros continuó in crescendo en 2008-2009 y la recesión se profundizó aún después de que la $i_{f f}$ fue reducida a su límite cero. El pánico financiero que se desencadenó a partir de la quiebra de Lehman Brothers contribuyó a profundizar el hiato entre las tasas de mercado y el límite cero de la $i_{f f}$, hiato que hace inoperante el mecanismo de transmisión de la política monetaria convencional para alcanzar la estabilidad de precios y la estabilidad financiera.

En los peores momentos de la crisis, la tasa de inflación general fue negativa, $-2 \%$ a mitad de 2009 , y la inflación subyacente fue menor (1.7\%) a la meta de $2 \%$. La inflación subyacente continuó disminuyendo hasta alcanzar $0.5 \%$ a fines de 2010 . El riesgo de que se repitiera la experiencia de deflación de Japón llegó a ser inminente, al tiempo que la tasa de desempleo incrementó de menos de 6\% en septiembre de 2008 a $10 \%$ en octubre de 2009 de acuerdo con datos del Bureau of Labor Statistics.

En este contexto de virtual estancamiento con deflación y agotamiento de las posibilidades de la política monetaria convencional, la Fed introdujo un nuevo 
marco de PMNC. Este nuevo enfoque reforzó los programas de liquidez, las facilidades de crédito de emergencia, las pruebas de estrés bancario y los acuerdos de swaps monetarios que la Fed aplicó como respuesta a la implosión financiera.

\section{Política monetaria no convencional: modus operandi y efectos}

La política monetaria no convencional es un esquema compuesto por dos elementos: el uso de la hoja de balance del banco central como instrumento de la política monetaria y el despliegue de estrategias de comunicación anticipada (forward guidance) para generar confianza en las acciones del banco central. En la PMNC la oferta monetaria es exógena y la tasa de interés es endógena. Formalmente puede expresarse así:

$$
\Delta M \equiv \Delta B M+D^{G}+C^{P}
$$

Donde $M$ es la oferta monetaria, $B M$ es la base monetaria, $D^{G}$ es la demanda de deuda o bonos del gobierno de los bancos comerciales y $C^{P}$ es el crédito al sector privado no bancario. La ecuación (5) significa que en la PMNC el control de la oferta monetaria depende del control de la $B M$, la $D^{G}$ y del $C^{P}$; la oferta de dinero determina a la demanda.

En teoría, los objetivos de la PMNC son: alcanzar la estabilidad de precios y la estabilidad financiera de manera integrada y simultánea; reducir las tasas de interés de largo plazo -la política monetaria convencional, en cambio, se enfoca en reducir la tasa de interés de corto plazo-, aumentando los precios de los bonos de largo plazo y reduciendo su rendimiento y la prima de riesgo de los bonos de más largo plazo; reducir las expectativas de inestabilidad de las tasas de interés de corto plazo futuras; reducir el riesgo de default; aumentar la liquidez en el mercado y estimular empleo y el producto (cf. Bernanke, 2013). La PMNC surte sus efectos a través de varios canales de transmisión que afectan a los precios de los activos y eventualmente a las tasas de interés de corto y largo plazo, a saber:

Primero, el canal del balance de portafolio: consiste en que mediante la compra de títulos del Tesoro el banco central altera la oferta y la demanda y, por ende, los precios relativos de activos sustitutos imperfectos entre sí. Se espera que este canal contribuya a estabilizar las tasas de interés y a crear un efecto riqueza favorable a la economía.

Segundo, el canal de liquidez: el retiro de títulos del Tesoro incrementa la liquidez del mercado de crédito, reduce o elimina la prima de riesgo y los costos de transacción en los mercados financieros, lo cual favorece las expectativas para los inversionistas.

Tercero, el canal de condiciones financieras: la expectativa de tasas de interés de largo plazo más bajas y estables, así como la mejora en las paridades de poder 
de compra debido a un tipo de cambio más competitivo, fortalece la posición en cuenta corriente de la balanza de pagos y la posición financiera neta de la economía con el exterior.

Cuarto, el canal de comunicación y confianza: la política de forward guidance contribuye a percibir los anuncios de política no convencional como señales que anticipan el futuro económico y financiero consistente con tasas de interés e inflación más estables.

La Fed adoptó un ambicioso programa de PMNC al término de la Gran Recesión y, principalmente, al momento de perder el control de la $i_{f f}$ (English et al., 2013). La figura 1 muestra la evolución de la $i_{f f}$. Es notable su caída drástica a partir de la crisis financiera.

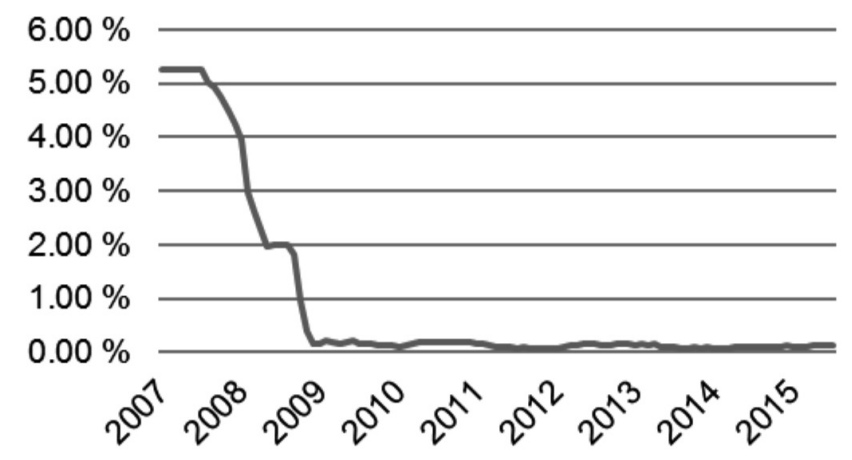

Figura 1. Tasa efectiva de fondos federales.

Fuente: Elaboración propia con estadísticas de la Reserva Federal (2015).

El uso de la hoja de balance adoptó la forma de sucesivas rondas de flexibilización cuantitativa o de crédito (QE por sus siglas en inglés) cuyo efecto principal discurre a través del canal de balance de portafolio. Las operaciones QE se realizan cuando la economía se encuentra en la trampa de liquidez y la tasa de interés de corto plazo no puede disminuir más mediante la política convencional. Con las operaciones QE el banco central adquiere grandes cantidades de activos financieros de largo plazo (por ejemplo, títulos respaldados por hipotecas (MBS = mortgage backed securities)), obligaciones de deuda colateralizada (CDO por sus siglas en inglés) en poder de los bancos. La Fed emite crédito a favor de las reservas de los bancos para adquirir bonos. Un enigma sobre el que a menudo inquiere el público es el siguiente: ¿de dónde surge el dinero para adquirir los activos financieros? La Fed tiene la capacidad de emitir dinero ex nihilo. Como consecuencia de las operaciones QE, la hoja de balance de la Fed se multiplicó por más de cuatro al ascender a 4.5 trillones de dólares (fig. 2). 


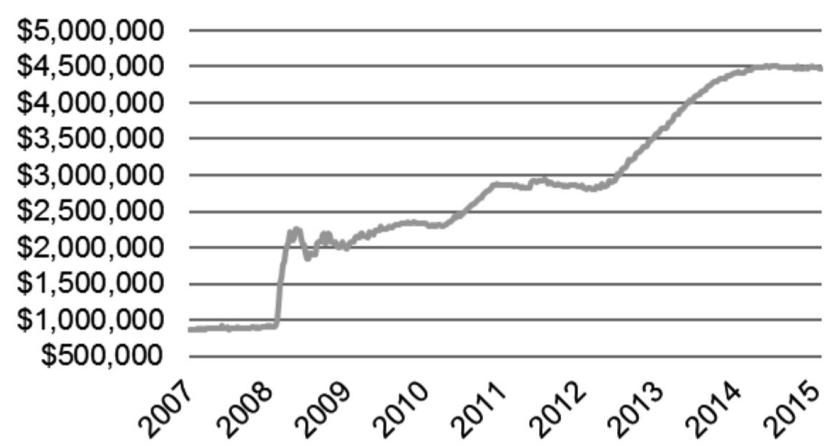

Figura 2. Hoja de Balance de la Reserva Federal (activo).

Fuente: Elaboración propia con estadísticas de la Reserva Federal (2015).

Estas transacciones QE producen un incremento proporcional en la base monetaria. El objetivo principal es disminuir la tasa de interés de largo plazo y así fijar una tasa de interés nominal de corto plazo. La Fed ha implementado tres rondas de flexibilización cuantitativa, QE1, QE2 y QE3, además de Operaciones Twist donde se realizan swaps entre bonos de corto y largo plazo con el mismo objetivo de enrocar bonos y dinero para incidir en la tasa de interés de largo plazo. La tabla 1 presenta la información calendarizada de las operaciones QE realizadas por la Fed.

El objetivo fundamental es aumentar el crédito para estimular el consumo, la inversión y el crecimiento económico. La Fed ofrece a los bancos comerciales precios adecuados por los títulos del Tesoro y los MBS y los bancos los intercambian por el exceso de reservas del banco central. La idea es que los bancos aumenten el crédito. El gran problema es que el crédito no ha aumentado, por varias razones; la principal de ellas es que en la economía norteamericana prevalece una recesión de hoja de balance en virtud de la cual los hogares, las pequeñas empresas y las corporaciones no financieras no demandan crédito... porque se encuentran en un proceso de desapalancamiento y su prioridad es ajustar su hoja de balance limpiándola de deuda contraída durante el auge de crédito que antecedió a la Gran recesión (cf. Koo, 2015).

Una segunda razón es que en un sistema económico donde la moneda es endógena, la oferta de crédito está regida por la demanda. Así, aunque la Fed inunde el mercado con exceso de reservas y continúe las operaciones QE, el estancamiento de la economía norteamericana no justificará un aumento en la demanda de crédito. 


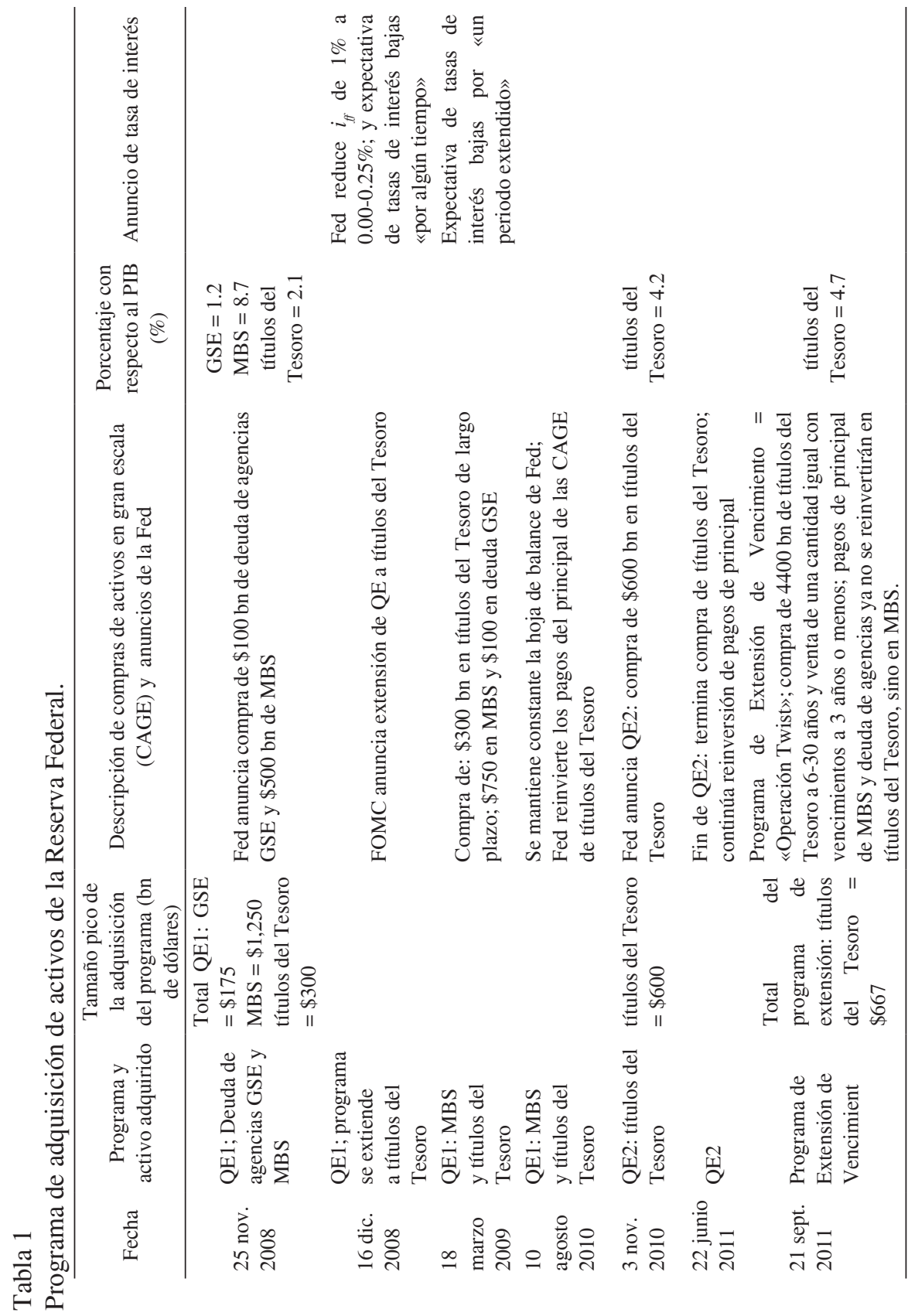




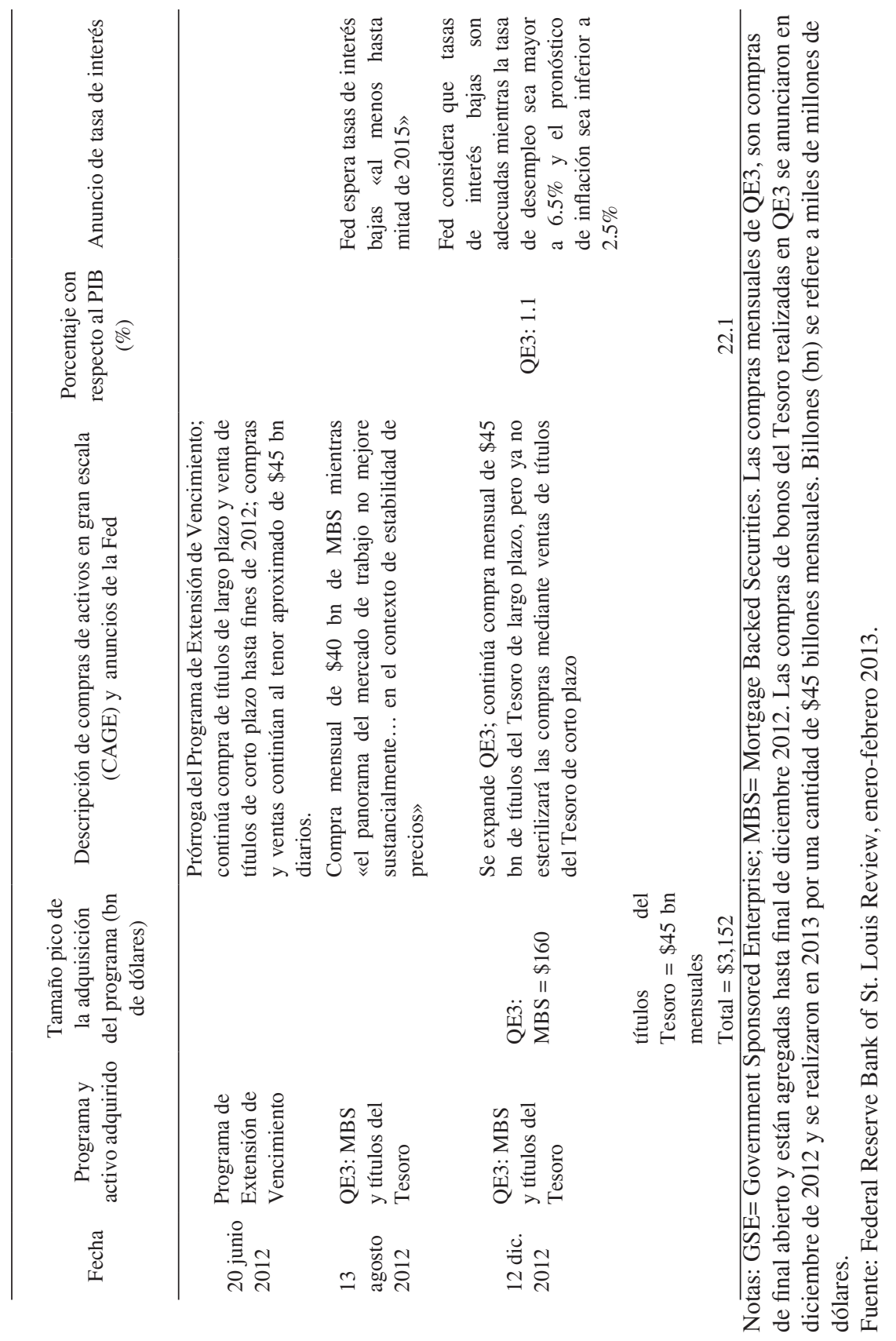


Finalmente, las masivas operaciones QE en rigor no son sino un enroque entre los depósitos de reservas en la Fed, por una parte, y los bonos del Tesoro y los títulos respaldados por las hipotecas (MBS); los fondos financieros se mueven entre las cuentas de ahorro que los bancos tienen registradas en la Fed (bonos del Tesoro) y las reservas de estos bancos en la propia Fed. Estas prestidigitaciones financieras pueden abatir las tasas de interés de largo plazo e incidir en la estructura de tasas de interés, generar rendimientos a los bancos, incluso mantener la tasa de inflación por debajo de la meta de la Fed (2\%), pero es cuestionable que la nueva PMNC per se necesariamente reactive a la economía.

\section{Algunos hechos estilizados}

¿Cuál ha sido el efecto de la PMNC y, en particular, de las colosales operaciones QE en la macroeconomía norteamericana? Después de la caída en picada del PIB en 2008, la economía se recuperó en 2009; y entre 2010 y 2015 creció a un ritmo lento sin alcanzar el $3 \%$ anual (fig. 3). En esta etapa la economía ha registrado el periodo más largo de crecimiento menor a 3\% desde la Gran Depresión.

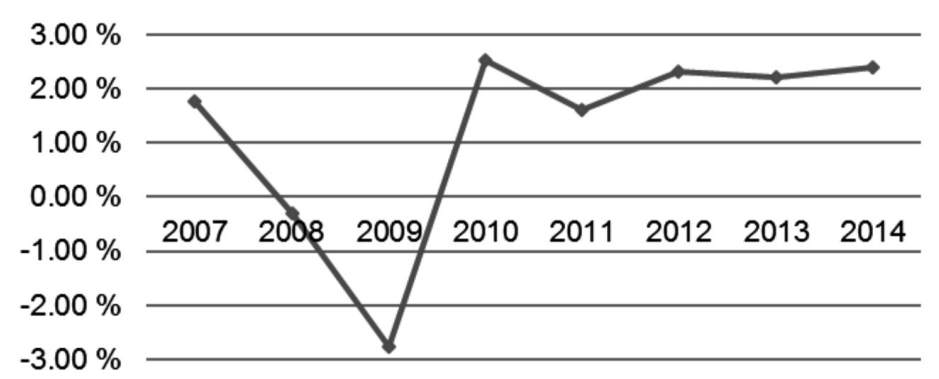

Figura 3. Crecimiento económico anual EE.UU.

Fuente: Elaboración propia con estadísticas del Banco Mundial (2015).

En segundo término, los temores de que las intervenciones de la Fed con sus operaciones QE detonarían una espiral inflacionaria no se han materializado. De acuerdo con el canon de la teoría cuantitativa del dinero, la nueva PMNC habría detonado una hiperinflación. Pero esto no ha ocurrido; la tasa de inflación subyacente se encuentra por debajo de la meta de la Fed; más aún, la probabilidad de deflación se encuentra más cercana a la realidad que la probabilidad de una hiperinflación (fig.4). 


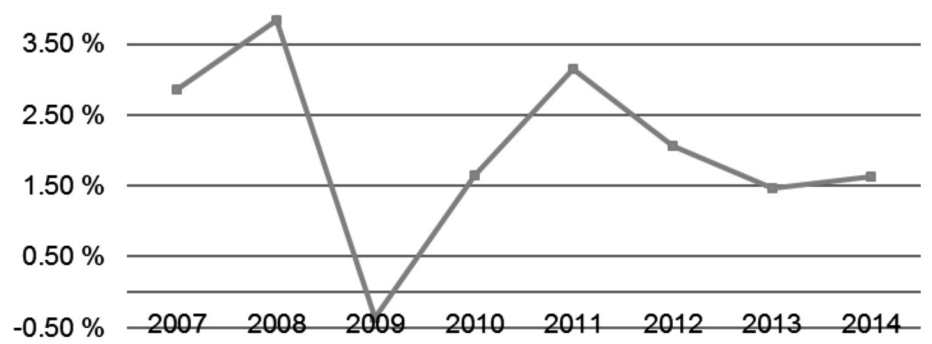

Figura 4. Inflación anual EE.UU.

Fuente: Elaboración propia con estadísticas del Bureau of Labor Statistics. (2015).

En tercer término, la Fed no ha alcanzado la meta de «empleo máximo». Las operaciones QE no han estimulado a Main Street, pero, por el contrario, la PMNC sí ha estimulado a Wall Street (fig. 5). En esto la PMNC se asemeja a la política monetaria convencional: ambas tienen el potencial de inducir auges financieros.

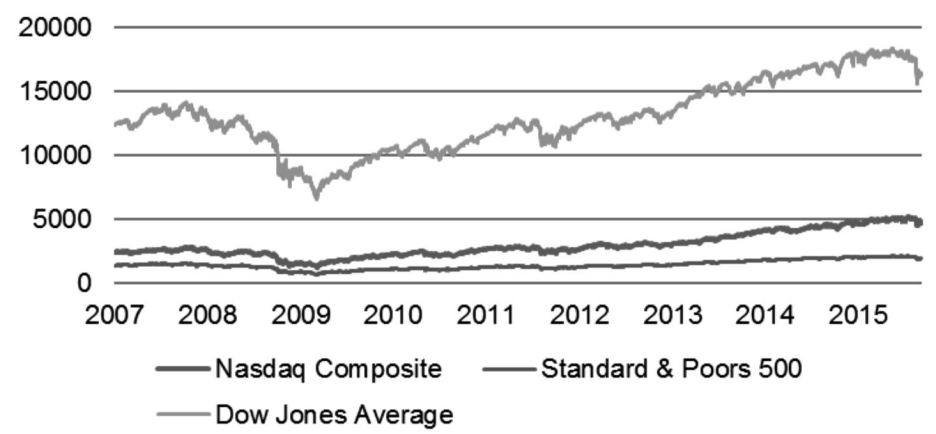

Figura 5. Índices de Valores de EE.UU.

Fuente: Elaboración propia con estadísticas de Bloomberg. (2015).

Las perspectivas hacia el 2018 indican que el futuro cercano y no tan cercano de la economía estadounidense no será muy distinto de lo que ya hemos observado desde 2009: la Fed pronostica un crecimiento económico lento entre 2015 y 2018 y aún más allá de 2018; la tasa de desempleo fluctuará alrededor de 5\% y la tasa de inflación se ubicará por debajo de la meta de $2 \%$, lo que significa que a pesar de la nueva PMNC es probable que la Fed no cumpla en parte su doble mandato de estabilidad de precios y máximo empleo. El único ítem en el que la Fed proyecta un cambio importante es en la tasa de interés de los fondos federales; la trayectoria de política de tasas de interés apropiada que proyecta muestra que la expectativa es que la tasa de interés aumentará en 2016, por lo menos se duplicará, y en 2017 y 2018 incrementará significativamente (tabla 2). Evidentemente, este cambio en la 
tasa de la política monetaria no necesariamente significaría un retorno a la «normalidad» (a la política monetaria convencional), pero si ocurre (o cuando ocurra) sin duda representará un choque de tasas de interés para la economía norteamericana (y, por extensión, para las economías latinoamericanas).

Tabla 2

Proyecciones económicas del Federal Reserve Board y de los presidentes de los bancos de la Federal Reserve. Septiembre 2015. Porcentajes.

\begin{tabular}{lccccc}
\hline \multirow{2}{*}{ Variable } & \multicolumn{5}{c}{ Tendencia central } \\
\cline { 2 - 6 } & 2015 & 2016 & 2017 & 2018 & Más largo plazo \\
\hline Variación en el PIB real & $2.0-2.3$ & $2.2-2.6$ & $2.0-2.4$ & $1.8-2.2$ & $1.8-2.2$ \\
Tasa de desempleo & $5.0-5.1$ & $4.7-4.9$ & $4.7-4.9$ & $4.7-5.0$ & $4.9-5.2$ \\
Inflación (GCP) & $0.3-0.5$ & $1.5-1.8$ & $1.8-2.0$ & 2.0 & N.D. \\
Inflación subyacente (GCP) & $1.3-1.4$ & $1.5-1.8$ & $1.8-2.0$ & $1.9-2.0$ & \\
& & & & & $3.3-3.8$ \\
Ruta de Política apropiada proyectada: & & & & $3.0-3.6$ &
\end{tabular}

Notas: la variación del PIB y las proyecciones de inflación son cambios porcentuales del cuarto trimestre del año previo al cuarto trimestre del año siguiente. Inflación (GCP) e inflación subyacente (GCP) son tasas de cambio porcentual del índice de precios del gasto en consumo personal y del gasto en consumo personal excluyendo alimentos y energía, respectivamente. Las proyecciones de tasa de desempleo son del promedio de la tasa de desempleo de la fuerza de trabajo civil correspondiente al cuarto trimestre del año indicado. Las proyecciones para el más largo plazo representan tasas a las que se espera que cada variable converja dada una política monetaria apropiada y en ausencia de ulteriores choques a la economía. Las proyecciones de la tasa de fondos federales son valores redondeados del punto medio del rango de objetivo apropiado proyectado de la tasa de fondos federales o del nivel objetivo apropiado proyectado de la tasa de fondos federales al final del año especificado o en el más largo plazo. La tendencia central excluye las tres proyecciones más altas y las tres más bajas para cada variable, para cada año. N.D. significa no disponible.

Fuente: Federal Reserve. Minutas del FOMC. (septiembre, 2015)

\section{Análisis empírico y resultados econométricos}

En esta parte analizamos cuantitativamente el efecto de la PMNC (QE), implementada por la FED, en la senda de crecimiento económico de EE.UU. Con este fin, se establece una relación empírica donde la dinámica económica $(g)$ es función del crédito privado $(C P)$, la hoja de balance $(H B)$, el tipo de cambio $(e)$ y la tasa de interés $(r)$ :

$$
g=f(C P, H B, e, r)
$$

Paralelamente, se plantea una especificación en la cual se relacionan la dinámica de la base monetaria y el nivel de producto para capturar de manera alterna el efecto de la PMNC, con lo cual tendremos: 


$$
g=f(C P, B M, e, r)
$$

Donde $B M$ denota la base monetaria. Con la inclusión de HB (compra de activos financieros de largo plazo) y BM se pretende capturar, sucesivamente, los efectos de la QE sobre el crecimiento económico. Para efectos empíricos, proponemos una especificación Autorregresiva de Rezagos Distribuidos (ARDL) de la forma:

$$
g_{t}=\sum_{j=1}^{p} \lambda_{j} g_{t-j}+\sum_{j=0}^{q-1} \theta_{j}^{\prime} X_{t-j}+u_{t}
$$

donde $g_{t}$ representa la dinámica de la economía, $X_{t}$ es un vector columna de $\mathrm{k}$ variables explicativas $(\mathrm{CP}, \mathrm{HB}, \mathrm{e}, \mathrm{r}) ; \lambda_{\mathbf{j}}$ y $\theta_{\mathbf{j}}^{\prime}$ son dos vectores de coeficientes y $u_{t}$ es un vector columna de perturbaciones aleatorias.

Si existe una relación de cointegración entre $\mathrm{g}_{\mathrm{t}} \mathrm{y} \mathrm{X}_{\mathrm{t}}$, el término de error sigue un proceso $\mathrm{I}(0)$. Por tanto, la relación de largo plazo deberá incorporar una ecuación de corrección de error, cuyo objetivo será incorporar la dinámica de corto plazo de las variables -influenciadas por las desviaciones respecto de la senda de equilibrioal comportamiento de largo plazo. Así, reescribimos la ecuación (7) como sigue:

$$
\Delta g_{t}=\beta_{0}+\beta^{\prime} H_{t}+\psi g_{t-1}+\sum_{j=1}^{p} \lambda_{j} \Delta g_{t-j}+\sum_{j=0}^{q-1} \theta_{j}^{\prime} \Delta X_{t-j}+\epsilon_{t}
$$

La ecuación (9) constituye la función de reacción de la dinámica económica sujeta a contraste empírico, donde $\Delta$ es el operador de primera diferencia; $\mathrm{g}_{\mathrm{t}}$ representa la evolución de la economía; $\psi$ el parámetro de corrección de error; Xi un vector con los determinantes de la ecuación de reacción (CP, HB, e, r); $\beta^{\prime}$ y $\theta_{j}^{\prime}$ los parámetros de largo y corto plazo, respectivamente; $\epsilon_{\mathrm{t}}$ el termino de error. Se espera que el coeficiente asociado al crédito, $\mathrm{CP}$, sea menor que 1 , con lo cual se confirmaría el efecto marginal de la flexibilización cuantitativa o de crédito («credit easing») sobre la inversión y la producción.

La expectativa respecto al coeficiente de $\mathrm{HB}$ es que sea menor que 1, lo cual indicaría que la política de QE no ha inducido un aumento en el crédito para estimular el consumo y la inversión, y con ello el crecimiento económico. En cuanto al impacto del tipo de cambio, e, se espera un efecto negativo sobre el nivel del producto, debido a que sus fluctuaciones pueden ejercer notables desfases de la demanda agregada, la inflación, el mercado crediticio y de capital.

Es importante acotar que el procedimiento ARDL (Pesaran y Shin, 1998; Pesaran, Shin y Smith, 2001) permite la combinación de variables con distinto orden 
de integración en el sistema, evitando con ello el problema asociado con el análisis de cointegración estándar que requiere las propiedades de estacionariedad de las variables; la metodología produce estimaciones no sesgadas de los coeficientes de largo plazo, a pesar de que las variables del sistema sean endógenas; los estimadores son eficientes y consistentes aun con muestras pequeñas.

Los datos comprenden series mensuales de enero de 2008 a Junio de 2015 para EE.UU. Como proxy de la dinámica económica se emplea el índice de producción industrial. Los datos sobre crédito corresponden al crédito privado, la hoja de balance se refiere al total de activos de la Reserva Federal; la información del tipo de cambio procede del índice ponderado del dólar respecto a las principales monedas y la tasa de interés es la de los fondos federales.

Primero, verificamos el orden de integración de las series mediante pruebas de raíz unitaria, Dickey-Fuller Aumentada (ADF) y Phillips-Perron (PP). El contraste de hipótesis sugiere, con un nivel de significancia del 5\%, que las variables son integradas I(1) en niveles y que sus primeras diferencias resultan procesos estocásticos $\mathrm{I}(0)$, excepto el índice de producción que sigue un proceso I(2) (fig. A1).

En segundo lugar, verificamos si entre las variables del sistema persiste una relación de equilibrio de largo plazo $\left(\mathrm{g}_{\mathrm{t}}, \mathrm{CP}_{\mathrm{t}}, \mathrm{HB}_{\mathrm{r}}, \mathrm{e}_{\mathrm{t}}, \mathrm{r}_{\mathrm{t}}\right)$, aplicamos el procedimiento de Pesaran, Shin y Smith (2001). La evidencia sugiere, según los estadísticos F y $\mathrm{t}^{4}$, la existencia de una relación de cointegración (fig. A.2). Las pruebas de diagnóstico no evidencian problemas de autocorrelación o heteroscedasticidad en los residuos.

La tabla 3 recoge las estimaciones de la relación de largo plazo, así como el coeficiente de velocidad de ajuste de las ecuaciones de reacción (6) y (7), respectivamente.

\footnotetext{
${ }^{4}$ La prueba se basa en los estadísticos F y t, mediante los cuales procuramos determinar la importancia de las variables rezagadas en niveles en un modelo de corrección de error univariado de la forma:

$$
\Delta g_{t}=\alpha_{0}+\alpha_{1} t+\phi g_{t-1}+\sum_{i=1}^{p} \Theta_{j} \Delta g_{t-i}+\sum_{i=1}^{q} \delta_{i} \Delta X_{t-i}+\epsilon_{t}
$$

El estadístico $\mathrm{F}$ contrasta la significancia estadística conjunta de las variables en niveles rezagadas, mientras que el estadístico t analiza la significancia estadística de la variable dependiente en niveles rezagada.
} 


\begin{tabular}{|c|c|c|c|c|c|c|c|c|}
\hline \multirow[b]{2}{*}{ País } & \multicolumn{3}{|c|}{ Dickey-Fuller Augmented } & \multicolumn{3}{|c|}{ Phillips-Perron } & \multirow{2}{*}{$\begin{array}{c}\# \\
\text { Rezago }\end{array}$} & \multirow[b]{2}{*}{$\mathrm{I}(\mathrm{d})$} \\
\hline & I & I y T & Sin I y $\mathrm{T}^{*}$ & I & I y $\mathrm{T}$ & Sin I y $T^{*}$ & & \\
\hline $\operatorname{lng}$ & 0.329 & $\begin{array}{c}-2.029 \\
{[0.2740]}\end{array}$ & $\begin{array}{c}-5.945 \\
{[0.0000]}\end{array}$ & 0.17 & $\begin{array}{c}-0.968 \\
{[0.7647]}\end{array}$ & $\begin{array}{c}-3.117 \\
{[0.1023]}\end{array}$ & 5 & $l(2) ; l(1)$ \\
\hline $\ln \mathrm{CP}$ & 1.842 & $\begin{array}{c}1.368 \\
{[0.9970]}\end{array}$ & $\begin{array}{c}-2.008 \\
{[0.5973]}\end{array}$ & 3.667 & $\begin{array}{c}2.505 \\
{[0.9991]}\end{array}$ & $\begin{array}{c}-1.178 \\
{[0.9150]}\end{array}$ & 3 & $l(1)$ \\
\hline $\ln H B$ & 1.920 & $\begin{array}{c}-2.840 \\
{[0.0527]}\end{array}$ & $\begin{array}{c}-3.364 \\
{[0.0564]}\end{array}$ & 1.855 & $\begin{array}{c}-2.238 \\
{[0.1927]}\end{array}$ & $\begin{array}{c}-2.575 \\
{[0.2914]}\end{array}$ & 3 & $l(1)$ \\
\hline $\ln e$ & 1.175 & $\begin{array}{c}-1.017 \\
{[0.7471]}\end{array}$ & $\begin{array}{c}-1.348 \\
{[0.8755]}\end{array}$ & 1.027 & $\begin{array}{c}-0.492 \\
{[0.8936]}\end{array}$ & $\begin{array}{c}-1.036 \\
{[0.9400]}\end{array}$ & 2 & $l(1)$ \\
\hline $\ln F R$ & -1.725 & $\begin{array}{c}-3.546 \\
{[0.0069]}\end{array}$ & $\begin{array}{c}-2.930 \\
{[0.1527]}\end{array}$ & -2.145 & $\begin{array}{c}-3.648 \\
{[0.0049]}\end{array}$ & $\begin{array}{c}-2.772 \\
{[0.2073]}\end{array}$ & 2 & $l(1)$ \\
\hline $\ln B M$ & 1.908 & $\begin{array}{c}-2.664 \\
{[0.0804]}\end{array}$ & $\begin{array}{c}-3.000 \\
{[0.1320]}\end{array}$ & 2.369 & $\begin{array}{c}-2.235 \\
{[0.1939]}\end{array}$ & $\begin{array}{c}-2.104 \\
{[0.5441]}\end{array}$ & 3 & $l(1)$ \\
\hline$\Delta \operatorname{lng}$ & -6.714 & $\begin{array}{c}-6.677 \\
{[0.0000]}\end{array}$ & $\begin{array}{c}-6.634 \\
{[0.0000]}\end{array}$ & -6.261 & $\begin{array}{c}-6.233 \\
{[0.0000]}\end{array}$ & $\begin{array}{c}-6.785 \\
{[0.0000]}\end{array}$ & 3 & $l(0)$ \\
\hline$\Delta \ln \mathrm{CP}$ & -2.408 & $\begin{array}{c}-3.039 \\
{[0.0314]}\end{array}$ & $\begin{array}{c}-4.165 \\
{[0.0051]}\end{array}$ & -5.869 & $\begin{array}{c}-7.086 \\
{[0.0000]}\end{array}$ & $\begin{array}{c}-8.439 \\
{[0.0000]}\end{array}$ & 2 & $l(0)$ \\
\hline$\Delta \ln \mathrm{HB}$ & -2.895 & $\begin{array}{c}-3.459 \\
{[0.0091]}\end{array}$ & $\begin{array}{c}-3.909 \\
{[0.0118]}\end{array}$ & -5.041 & $\begin{array}{c}-5.150 \\
{[0.0000]}\end{array}$ & $\begin{array}{c}-5.177 \\
{[0.0000]}\end{array}$ & 7 & $l(0)$ \\
\hline$\Delta l n \mathrm{e}$ & -3.519 & $\begin{array}{c}-3.636 \\
{[0.0051]}\end{array}$ & $\begin{array}{c}-3.676 \\
{[0.0240]}\end{array}$ & -6.127 & $\begin{array}{c}-6.179 \\
{[0.0000]}\end{array}$ & $\begin{array}{c}-6.264 \\
{[0.0000]}\end{array}$ & 3 & $l(0)$ \\
\hline$\Delta \ln F R$ & -5.475 & $\begin{array}{c}-5.591 \\
{[0.0000]}\end{array}$ & $\begin{array}{c}-5.991 \\
{[0.0000]}\end{array}$ & -6.211 & $\begin{array}{c}-6.296 \\
{[0.0000]}\end{array}$ & $\begin{array}{c}-6.583 \\
{[0.0000]}\end{array}$ & 1 & $l(0)$ \\
\hline$\Delta \ln B M$ & -3.989 & $\begin{array}{c}-4.626 \\
{[0.0001]}\end{array}$ & $\begin{array}{c}-4.983 \\
{[0.0002]}\end{array}$ & -4.663 & $\begin{array}{c}-5.094 \\
{[0.0000]}\end{array}$ & $\begin{array}{c}-5.263 \\
{[0.0001]}\end{array}$ & 2 & $l(0)$ \\
\hline
\end{tabular}

Figura A1. Orden de integración. Prueba de raíz unitaria.

El cuadro reporta el valor del estadístico Z(t), así como el valor-p correspondiente (entre corchetes). I: Intercepto, I y T: intercepto y tendencia.

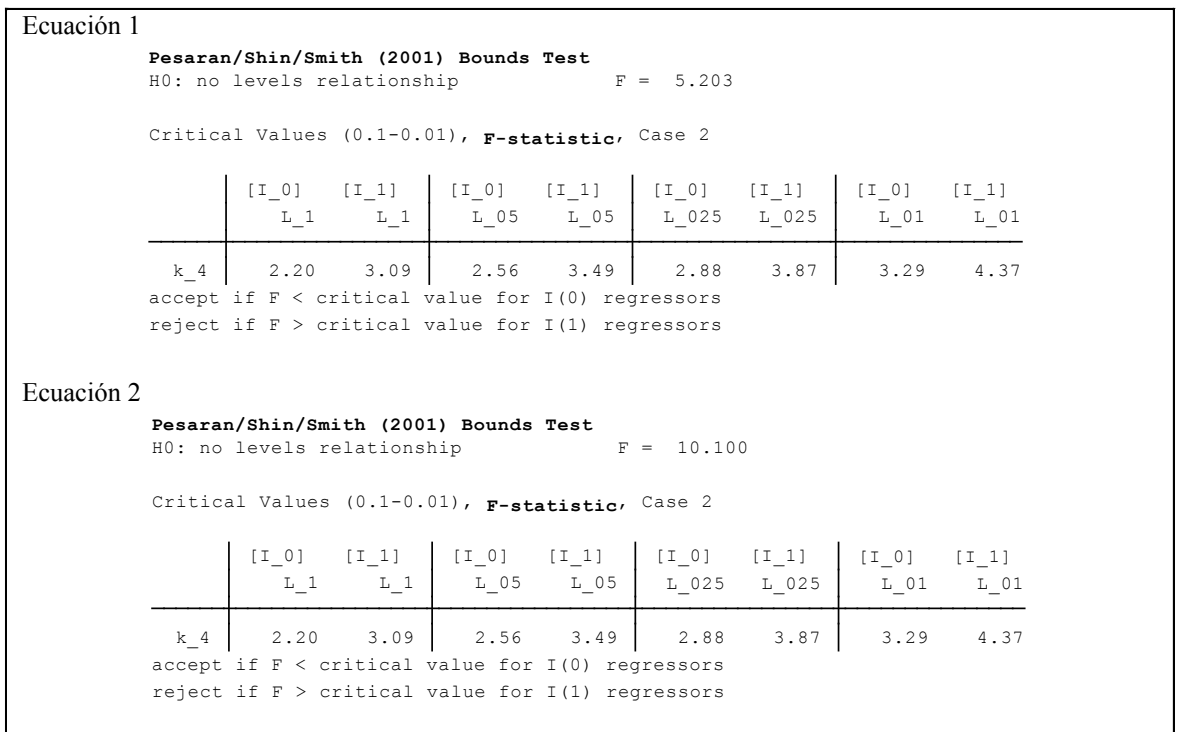

Figura A2. Prueba de Cointegración. Procedimiento de Pesaran, Shin y Smith (2001). 
Tabla 3

Dinámica económica y política monetaria no convencional.

\begin{tabular}{|c|c|c|c|c|c|c|c|}
\hline \multicolumn{8}{|c|}{ Relación de equilibrio } \\
\hline $\begin{array}{l}\text { Variables } \\
\text { del Sistema }\end{array}$ & $\begin{array}{c}\text { Constante } \\
\mathrm{C} \\
\end{array}$ & $\begin{array}{c}\text { Crédito } \\
\text { Privado } \mathrm{CP}_{t}\end{array}$ & $\begin{array}{c}\text { Hoja de } \\
\text { Balance } \mathrm{HB}_{\mathrm{r}}\end{array}$ & $\begin{array}{c}\text { Base Monetaria } \\
\mathrm{BM}_{t}\end{array}$ & $\begin{array}{l}\text { Tipo de } \\
\text { cambio e }\end{array}$ & $\begin{array}{l}\text { Tasa de } \\
\text { Interés } r_{t}\end{array}$ & $g_{t-1}$ \\
\hline Ecuación (6) & $\begin{array}{c}5.5132 \\
{[0.001]^{*}}\end{array}$ & $\begin{array}{l}0.0263 \\
{[0.932]}\end{array}$ & $\begin{array}{c}0.3137 \\
{[0.030]^{*}}\end{array}$ & 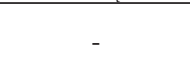 & $\begin{array}{c}-0.6059 \\
{[0.006]^{*}}\end{array}$ & $\begin{array}{c}0.0747 \\
{[0.051]^{* *}}\end{array}$ & $\begin{array}{c}-0.0893 \\
{[0.030]^{*}}\end{array}$ \\
\hline Ecuación (7) & $\begin{array}{c}4.7028 \\
{[0.000]^{*}}\end{array}$ & $\begin{array}{l}0.0359 \\
{[0.846]}\end{array}$ & 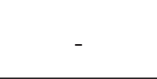 & $\begin{array}{c}0.2528 \\
{[0.001]^{*}}\end{array}$ & $\begin{array}{l}-0.3719 \\
{[0.000]^{*}}\end{array}$ & $\begin{array}{c}0.0687 \\
{[0.007]^{*}}\end{array}$ & $\begin{array}{l}-0.1486 \\
{[0.001]^{*}}\end{array}$ \\
\hline
\end{tabular}

*Significativo al 5\%

$* *$ Significativo al $10 \%$

Valor-p entre paréntesis

Fuente: Elaboración del autor con base en una especificación ARDL.

La estimación de ambas ecuaciones presenta consistencia en términos del sentido y significancia de los parámetros obtenidos (fig. A3).

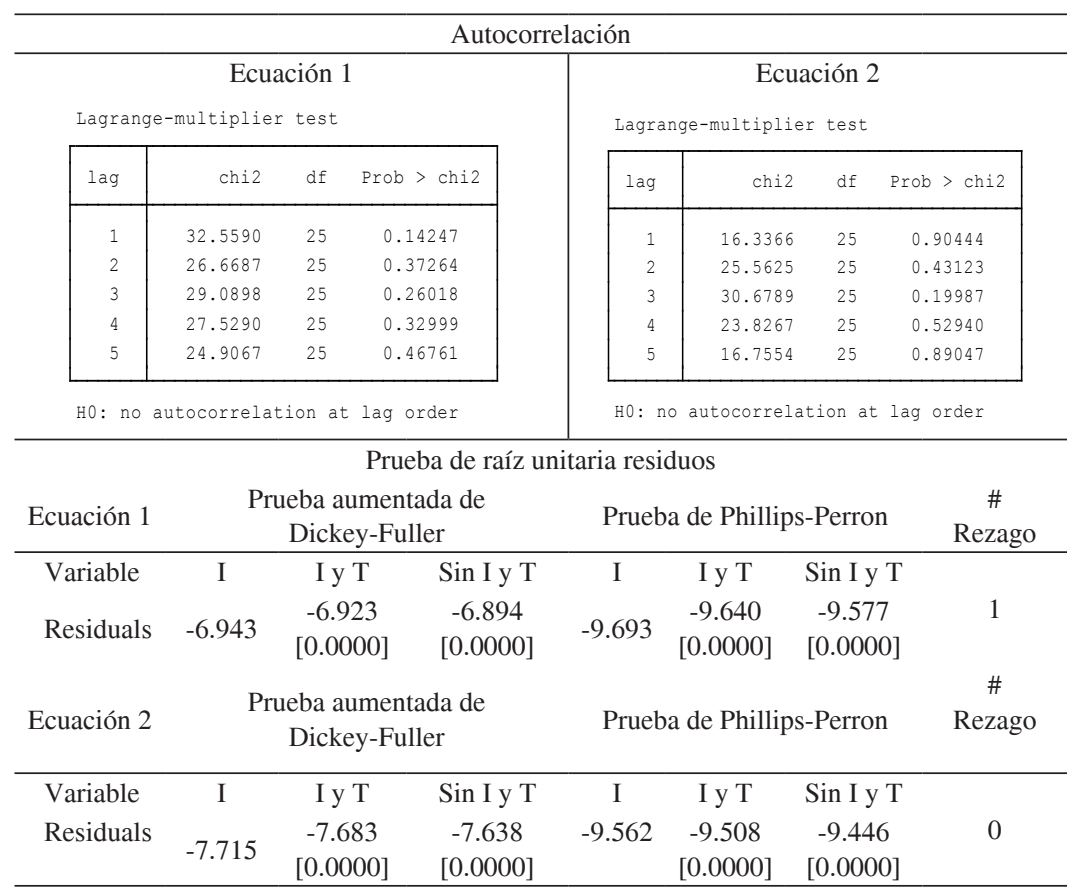

Figura A3. Pruebas de consistencia

La figura reporta el valor del estadístico Z(t), así como el valor-p correspondiente [entre corchetes]. I: Intercepto, I y T: intercepto y tendencia.

Significativo al $5 \%$

Significativo al $10 \%$. 
En principio, la condición de equilibrio de la ecuación 1 queda expresada como:

$$
g=5.51+0.02 C P+0.31 H B-0.61 e+0.07 r
$$

De acuerdo con los resultados obtenidos, el coeficiente del crédito carece de significancia estadística, lo cual sugiere que el crédito no ha tenido un efecto significativo en la reactivación de la actividad económica de EE.UU. Entre otras razones, porque la política de QE no está impulsando la oferta de crédito, y éste no alienta las expectativas de inversión, en parte debido al proceso de desapalancamiento de los agentes económicos y de recesión de hoja de balance (Koo, 2015).

Por otro lado, si bien los resultados muestran que ante un aumento en $1 \%$ de $\mathrm{HB}$ el nivel de producto aumenta en $0.31 \%$, el efecto es marginal e insuficiente en términos de: estabilizar las tasas de interés, crear un efecto riqueza, mejorar la liquidez del mercado de crédito, las expectativas del mercado financiero y mejorar la posición en cuenta corriente de la balanza de pagos y la posición financiera neta de la economía con el exterior (fig. A4).

\begin{tabular}{|c|c|c|c|c|c|c|}
\hline \multicolumn{7}{|c|}{$\begin{array}{l}\text { Ecuación } 1 \\
\text { a ardl Inipi Inicred lnihb lnitc lnifr, maxc }(2500) \text { ec aic res }\end{array}$} \\
\hline \multicolumn{7}{|l|}{ ARDL regression } \\
\hline $\begin{array}{l}\text { Sample: } 2008 \mathrm{~m} \\
\text { Number of obs } \\
\text { Log likelihood } \\
\text { R-squared } \\
\text { Adj R-squared } \\
\text { Root MSE }\end{array}$ & $\begin{array}{l}5-2015 \mathrm{~m} 7 \\
=87 \\
=323.40165 \\
=.51654402 \\
=.45293139 \\
=.00629115\end{array}$ & & & & & \\
\hline D.1nipi & Coef. & Std. Err. & t & $P>|t|$ & [95\% Conf. & Interval] \\
\hline $\begin{array}{l}\text { ADJ } \\
\quad \text { Inipi } \\
\text { L1. }\end{array}$ & -.0893413 & .0404958 & -2.21 & 0.030 & -.1699956 & -.008687 \\
\hline LR & & & & & & \\
\hline $\begin{array}{r}\text { Inicred } \\
\text { 1ninb } \\
\text { 1nitc } \\
\text { 1nifr } \\
\text { _cons }\end{array}$ & $\begin{array}{r}.0262974 \\
.3138518 \\
-.6059302 \\
.0747467 \\
5.513207\end{array}$ & $\begin{array}{l}.3051085 \\
.1419604 \\
.2160328 \\
.0377127 \\
1.401044\end{array}$ & \begin{tabular}{r|}
0.09 \\
2.21 \\
-2.80 \\
1.98 \\
3.94
\end{tabular} & $\begin{array}{l}0.932 \\
0.030 \\
0.006 \\
0.051 \\
0.000\end{array}$ & $\begin{array}{r}-.5813787 \\
.0311131 \\
-1.036197 \\
-.0003646 \\
2.722785\end{array}$ & $\begin{array}{r}.6339735 \\
.5965904 \\
-.1756637 \\
.149858 \\
8.303628\end{array}$ \\
\hline $\begin{array}{r}\text { Inipi } \\
\text { LD. }\end{array}$ & .1770297 & .1229564 & 1.44 & 0.154 & -.0678591 & .4219186 \\
\hline $\begin{array}{l}\text { Ininb } \\
\text { D1. } \\
\text { LD. } \\
\text { L2D. }\end{array}$ & $\begin{array}{r}.0128129 \\
-.0198835 \\
-.0328473\end{array}$ & $\begin{array}{l}.0183681 \\
.0168049 \\
.0148526\end{array}$ & $\begin{array}{r}0.70 \\
-1.18 \\
-2.21\end{array}$ & $\begin{array}{l}0.488 \\
0.240 \\
0.030\end{array}$ & $\begin{array}{l}-.0237704 \\
-.0533533 \\
-.0624287\end{array}$ & $\begin{array}{r}.0493962 \\
.0135864 \\
-.0032658\end{array}$ \\
\hline $\begin{array}{c}\text { Initc } \\
\text { D1. }\end{array}$ & .0632424 & .0461042 & 1.37 & 0.174 & -.028582 & .1550669 \\
\hline
\end{tabular}




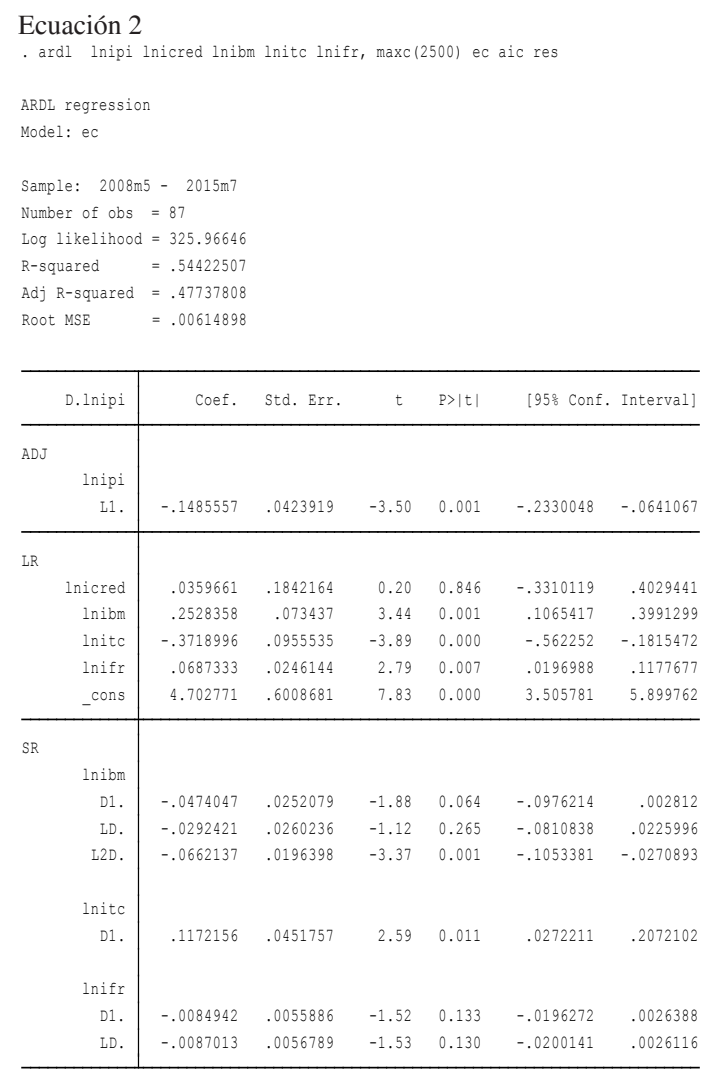

Figura A4. Estimación ecuación de reacción del crecimiento.

En cuanto al índice del tipo de cambio, se observa una relación negativa con la dinámica económica; un aumento (depreciación) en $1 \%$ del tipo de cambio genera una contracción del producto en $\mathbf{0 . 6 0 \%}$. Este resultado parece confirmar que en un entorno de déficit en balanza de pagos, de contracción de demanda agregada, inelasticidad de la oferta y redistribución del ingreso (transición del sector privado al sector público), la depreciación del tipo de cambio generará efectos negativos sobre el nivel de actividad económica.

Por otra parte, las estimaciones confirman una relación positiva y estadísticamente significativa entre la tasa de interés de los fondos federales y el nivel de producción. Así un aumento en $1 \%$ de la tasa de interés, genera una expansión de $0.07 \%$ sobre el nivel del producto. En principio, este resultado revela el débil impacto de la tasa de interés sobre las expectativas de inversión/ahorro y, por ende, 
sobre la demanda efectiva. El límite cero de la tasa de interés nominal restringe la efectividad de la política monetaria para afectar el nivel de empleo y del producto, incluso con el aumento de la oferta monetaria mediante el mecanismo de flexibilización cuantitativa o de crédito orientado a reactivar el mercado de crédito, la inversión y la demanda agregada.

En la estimación del modelo (7) destaca el sentido de asociación de la BM con el nivel de producto, ya que converge con los resultados encontrados y con la evidencia de la elasticidad de la HB. Así, ante un incremento de $1 \%$ en la BM se genera un impulso marginal en el nivel de producción de alrededor de $0.25 \%$.

\section{Conclusiones}

En el presente artículo analizamos los fundamentos y los límites de la política monetaria no convencional practicada por la Fed durante la post-Gran Recesión para contener el pánico financiero, evitar el riesgo de deflación y alentar la endeble recuperación de la economía.

Después de la discusión teórica sobre las implicaciones de la trampa de liquidez, la política de tasa de interés cero y la revisión sucinta de los hechos estilizados relevantes y los pronósticos de la inflación, el crecimiento, el desempleo y la evolución del mercado de valores, realizamos una estimación econométrica de la relación empírica entre la PMNC y la macrodinámica de la economía norteamericana. Estimamos la dinámica económica $(g)$ en función del crédito privado $(C P)$, la hoja de balance $(H B)$, el tipo de cambio (e) y la tasa de interés $(r)$. La evidencia empírica muestra que la PMNC no ha contribuido a acelerar el crecimiento de la economía de EE.UU., quid erat demonstrandum!

Aunque, como sugieren otros autores (Chung et al., 2011), es probable que la PMNC haya contribuido a evitar la deflación. Este tema, así como el potencial impacto sobre las economías latinoamericanas del eventual retorno de la Fed a la política monetaria convencional y las implicaciones de la PMNC para la teoría monetaria, representan líneas de investigación promisorias que se derivan del presente análisis para futuras pesquisas.

\section{Referencias}

Banco Mundial. (2015). Perspectivas de la economía mundial. Washington D.C.

Bernanke, B. S., Laubach, T, Mishkin, F. S., y Posen, A. (1999). Inflation Targeting: lessons from the international experience. Princeton: Princeton University Press. 
Bernanke, B. S. (2012). Opening Remarks: Monetary Policy since the Onset of the Crisis. Economic Policy Symposium auspiciado por the Federal Reserve Bank of Kansas City, "The Changing Policy Landscape". Jackson Hole, Wyoming. Agosto 31. Disponible en http://www.federalreserve.gov/newsevents/speech/bernanke20120831a.htm. (Consultado el 25 de julio de 2015).

Bernanke, B. S. (2013). The Federal Reserve and the Financial Crisis. Princeton: Princeton University Press. Bloomberg. (2015). Base de Datos. Nueva York.

Board of Governors of the Federal Reserve System. (2014). Policy Normalization Principles and Plans. Monetary Policy Releases. Septiembre 17. Disponible en http://www.federalreserve. gov/newsevents/press/monetary/20140917c.htm. (Consultado el 25 de julio de 2015).

Bureau of Labor Statistics. (2015). Consumer Index Price. Washington D.C.

Capraro, S. y Perrotini, I. (2012). Intervenciones Cambiarias Esterilizadas, Teoría y Evidencia: El Caso de México. Contaduría y Administración, 57 (2), 11-44.

Chung, H., Laforte, J-P., Reifschneider, D. y Williams, J. C. (2011). Have We Underestimated the Likelihood and Severity of Zero Lower Bound Events? Working Paper 2011-01, Federal Reserve Bank of San Francisco. Disponible en http://www.frbsf.org/publications/economics/papers/2011/wp11-01bk.pdf. (Consultado el 25 de julio de 2015).

English, W. B., López-Salcido, J. D., y Tetlow, R. (2013). The Federal Reserve's Framework for Monetary Policy -Recent Changes and New Questions. Washington, D.C. International Monetary Fund.

Fisher, I. (1907). The Rate of Interest. Nueva York: Macmillan.

García, A. y Perrotini, I. (2014). El modus operandi del Nuevo Consenso Macroeconómico en Brasil, Chile y México. Revista Problemas del Desarrollo, 45 (179), 35-63.

Goodhart, C. A. E. (2009). The Continuing Muddle of Monetary Theory: A Steadfast Refusal to Face Facts. Economica, 76, 821-830.

Keynes, J. M. ([1936] 1964). The General Theory of Employment, Interest, and Money. Nueva York: Harcourt Brace Jovanovich Publishers.

Koo, R. C. (2015). The Escape from Balance Sheet Recession and the QE Trap: A Hazardous Road for the World Economy. Singapur: John Wiley and Sons.

Minsky, H. P. (1975). John Maynard Keynes. Nueva York: Columbia University Press.

Minsky, H. P. (1986). Stabilizing an Unstable Economy. New Haven: Yale University Press.

Pérez Caldentey, E. (2015). Una coyuntura propicia para reflexionar sobre los espacios para el debate y el diálogo entre neoestructuralismo y las corrientes heterodoxas. En A. Bárcena y A. Prado (Eds.), Neoestructuralismo y corrientes heterodoxas en América Latina y el Caribe a inicios del siglo XXI, 35-81. Santiago de Chile: CEPAL.

Pesaran M. H. y Shin, Y. (1998). Generalized impulse response analysis in linear multivariate models. Economics Letters, 58 (1), 17-29.

Pesaran M. H., Shin, Y. y Smith, R. J. (2001). Bounds testing approaches to the analysis of level relationships. Journal of Applied Econometrics, (16), 289-326.

Setterfield, M. (2005). Central bank behavior and the stability of macroeconomic equilibrium: a critical examination of the "New Consensus", en P. Arestis, M. Baddeley y J. McCombie (Eds.), The new monetary policy: Implications and relevance, 23-49. Cheltenham, U.K. Edward Elgar.

Svensson, L. (1998). Open-economy inflation targeting. NBER Working Papers, no. 6, 545, Massachusetts, EE.UU. NBER, mayo, 1-53.

Taylor, J. (1993). Discretion versus policy rules in practice. Carnegie- Rochester Conference Series on Public Policy, 39, 195-214.

Woodford, M. (2003). Interest and prices: Foundations of a theory of monetary policy. Princeton: Princeton University Press. 